\title{
ON LOCALLY AND GLOBALLY CONFORMAL KÄHLER MANIFOLDS
}

BY

IZU VAISMAN

\begin{abstract}
Some relations between the locally conformal Kähler (l.c.K.) and the globally conformal Kähler (g.c.K.) properties are established. Compact l.c.K. manifolds which are not g.c.K. do not have Kähler metrics. l.c.K. manifolds which are not g.c.K. are analytically irreducible. Various curvature restrictions on l.c.K. manifolds imply the g.c.K. property. Total spaces of induced Hopf fibrations are 1.c.K. and not g.c.K. manifolds.

ConJeCture. A compact l.c.K. manifold which is not g.c.K. has at least one odd odd-dimensional Betti number.
\end{abstract}

1. Introduction. The purpose of this note is to discuss some relations between locally conformal Kähler (l.c.K.) and globally conformal Kähler (g.c.K.) metrics. Various results concerning this subject are already known [1] and [10]-[13]. Here we shall establish the interesting fact that any l.c.K. metric on a compact Käher manifold is a g.c.K. metric, and we shall discuss some new curvature criteria for an 1.c.K. metric to be g.c.K. Furthermore, we shall prove that a reducible l.c.K. manifold must be Kähler, and we shall discuss some examples of l.c.K. manifolds which are not g.c.K. I am glad to thank here S. I. Goldberg for the useful discussions we had on these matters.

2. I.c.K. metrics on compact Kähler manifolds. Let us mention first that, in discussing our subject, we shall use the notation and definitions of [10]-[14]. I.e. we shall denote: by $M$ an l.c.K. manifold $\left(\operatorname{dim}_{C} M=n \geqslant 2\right)$; by $g$ its metric; by $\left\{U_{\alpha}\right\}$ an open covering of $M$ endowed with $C^{\infty}$-functions $\sigma_{\alpha}: U_{\alpha} \rightarrow R$ such that $\tilde{g}_{\alpha}=$ $e^{-\sigma_{\alpha} g}$ are Kähler metrics; by $J$ the complex structure; by $\nabla$ the Levi-Civita connection of $g$; by $\tilde{\nabla}$ the Weyl connection of $g$, i.e., the Levi-Civita connection of $\tilde{g}_{\alpha}$; by $\omega\left(=d \sigma_{\alpha}\right)$ the Lee form of $M$, which is closed and satisfies $d \Omega=\omega \wedge \Omega$, where $\Omega$ is the fundamental form of $M$; and by $B$ the Lee field, i.e., the contravariant vector field associated to $\omega$.

The result stated in the Introduction, and which we shall be proving here, is

THEOREM 2.1. The compact l.c.K. manifold $(M, J, g)$ is g.c.K. iff $(M, J)$ bears some Kähler metric $\gamma$.

Proof. The stated condition is obviously a necessary one.

In order to prove that it is also sufficient, we shall start by the consideration of

Received by the editors November 28, 1979.

1980 Mathematics Subject Classification. Primary 53C55.

(C) 1980 American Mathematical Society $0002-9947 / 80 / 0000-0563 / \$ 03.50$ 
the anti-Lee form $\theta=\omega \circ J$. (Note that it has an associated anti-Lee field $A=$ $-J B$.) If we put $\omega=\omega^{\prime}+\omega^{\prime \prime}$, where the components are of the complex type $(1,0)$ and $(0,1)$, respectively, we have $\theta=\sqrt{-1}\left(\omega^{\prime}-\omega^{\prime \prime}\right)$ and $d \theta=2 \sqrt{-1} d^{\prime \prime} \omega^{\prime}$, which implies that $d \theta$ is a real exact form of type $(1,1)$.

Hence, if the Kähler metric $\gamma$ exists, we have (e.g., [5, Lemma 2, p. 13])

$$
d \theta=2 \sqrt{-1} d^{\prime} d^{\prime \prime} \ln f
$$

for some $C^{\infty}$ globally defined positive function $f: M \rightarrow R$, i.e., we have

$$
d^{\prime \prime} \omega^{\prime}=d^{\prime} d^{\prime \prime} \ln f \text {. }
$$

Let us now take the metric $h=f g$ on $M$. It is obviously l.c.K., and it is easy to see that its Lee form is $\tilde{\omega}=\omega+d \ln f$ and, because of $(2.2)$, its (1,0)-component $\tilde{\omega}^{\prime}$ satisfies

$$
d^{\prime \prime} \tilde{\omega}^{\prime}=0
$$

Now by using local complex coordinates $z^{j}, z^{\bar{j}}=\bar{z}^{j}$, we have some expression $\tilde{\omega}^{\prime}=\lambda_{j} d z^{j}$, where, by (2.3), $\lambda_{j}$ are complex analytic functions. In turn, and since $\tilde{g}_{\alpha}$ are Kähler metrics, this is equivalent to

$$
\tilde{\nabla}_{\bar{j}} \lambda_{k}=0 \text {. }
$$

If we shall be using the existing conformal relation between $h$ and $\tilde{g}_{\alpha}$, we shall see that (2.4) is equivalent to

$$
D_{\bar{j}} \lambda_{k}=-\lambda_{k} \lambda_{j}+h_{k j} h^{\bar{p} h} \lambda_{\bar{p}} \lambda_{h} \quad\left(\lambda_{\bar{j}}=\bar{\lambda}_{j}\right),
$$

where $D$ denotes the Levi-Civita connection of $h$.

Furthermore, (2.5) implies

$$
\delta_{h} \tilde{\omega}^{\prime}=-((n-1) / 2)|\tilde{\omega}|_{h}^{2},
$$

where the index $h$ indicates that the operations are performed with respect to the metric $h$.

Now, by integrating (2.6) over $M$, we obtain $\tilde{\omega}=0$, which means that $h$ is a Kähler metric, and the theorem is proven.

Corollary 2.2. A compact l.c.K. manifold which is not g.c.K. possesses no Kähler metric.

ReMARKs. (1) As a matter of fact, the proof of Theorem 2.1 holds if the hypothesis of the existence of the Kähler metric $\gamma$ is replaced by the weaker hypothesis that the $d^{\prime} d^{\prime \prime}$-cohomology class defined by $d \theta$ vanishes. Particularly, if $d \theta=0$ (i.e., $\omega^{\prime}$ is a holomorphic 1 -form) and $M$ is compact, $g$ is a Kähler metric.

(2) Theorem 2.1 and Corollary 2.2 do not hold in the noncompact case. This follows from the existence of an l.c.K. metric on $T_{c}^{1} \times C^{n-1}$ which is not g.c.K. and which has been constructed in [10].

We would like to add the following comments. In view of Theorem 2.1 , the two problems of (1) characterizing the 1.c.K. manifolds which are g.c.K., and (2) characterizing the 1.c.K. manifolds which bear a Kähler metric [13] are equivalent in the compact case. Furthermore, the conjecture that a compact l.c.K. manifold, which satisfies the Kählerian topological restrictions, possesses a Kähler metric 
[11], must be reformulated by conjecturing that, as a matter of fact, the given metric must be g.c.K. Let us note that this result will be implied by the proof of

CONJECTURE. A compact l.c.K. manifold which is not g.c.K. has at least one odd odd-dimensional Betti number.

This last conjecture has been proven by Kashiwada and Sato [2] in the case of the 1.c.K. manifolds with parallel Lee form. It is the first Betti number of the manifold which must be odd in this case.

Moreover, we can see that the conjecture holds for l.c.K. manifolds of complex dimension $n=2$, i.e., we have

Proposition 2.3. A compact l.c.K., but not g.c.K., manifold $M$ of complex dimension 2 has an odd first Betti number.

Proof. Let us assume that the manifold $M$ of the proposition has an even first Betti number $b_{1}=2 q$. Then, it is proven in Kodaira [4] that there are $q$ linearly independent closed holomorphic 1 -forms $\phi_{1}, \ldots, \phi_{q}$ such that the cohomology classes of these forms and of their complex conjugates form a basis in $H^{1}(M, C)$.

Hence, we have for the Lee form of $M$

$$
\omega=\alpha_{1} \phi_{1}+\cdots+\alpha_{q} \phi_{q}+\beta_{1} \bar{\phi}_{1}+\cdots+\beta_{q} \bar{\phi}_{q}+d \varphi
$$

for some constants $\alpha, \beta$ and some function $\varphi$. It follows that

$$
\omega^{\prime}=\alpha_{1} \phi_{1}+\cdots+\alpha_{q} \phi_{q}+d^{\prime} \varphi,
$$

and $d \theta=-2 \sqrt{-1} d^{\prime} d^{\prime \prime} \varphi$. By Remark (1), this implies that $M$ is g.c.K., which is not the case.

Hence, under the hypotheses of Proposition 2.3, we must have $b_{1}(M)$ odd. Q.E.D.

We see thereby that compact two-dimensional 1.c.K. and not g.c.K. manifolds can exist only in the classes VI and VII of Kodaira's classification of surfaces [4].

3. Curvature criteria for g.c.K. metrics. It has been proven in [12] and [1] that, under some restrictions on the Riemann curvature tensor of $g$, a compact l.c.K. manifold is, in fact, Kähler. For instance, I would like to note that the result of Theorem 2.1 of [1] follows from

Proposition 3.1. Assume that $M$ is a compact l.c.K. manifold such that, for any two holomorphic 2-planes defined by the unit vectors $X, Y$, respectively, the holomorphic bisectional curvature is related to the sectional curvatures by the inequality

$$
R(X, J X, Y, J Y) \geqslant R(X, Y, X, Y)+R(X, J Y, X, J Y) .
$$

(The convention sign for $R$ is that of [3].) Then $M$ must be a Kähler manifold.

The proof follows from the fact that (3.1) implies the inequality assumed in [1], and it consists of summing up (3.1) over all the pairs of an orthonormal basis $\left\{E_{i}, J E_{i}\right\}$.

It is also noteworthy that the inequality assumed in Theorem 2.1 of [1] holds in the case of an 1.c.K. manifold of constant sectional curvature $k \leqslant 0$, which implies 
Proposition 3.2. A compact l.c.K. manifold of constant sectional curvature must be a flat Kähler manifold.

This is a consequence of the above-mentioned argument if $k \leqslant 0$, and it follows from the fact that the high-dimensional spheres do not have complex structures (dimension six excluded?) if $k>0$.

Now, the purpose of this section is to produce some curvature criteria for the g.c.K. property by using the unitary connection of $M[8]$ instead of the Levi-Civita connection.

The unitary connection $\nabla_{(c)}$ of a Hermitian manifold is defined by the conditions [3, Chapter IX, Proposition 10.3]

$$
\nabla_{(c)} J=0, \quad \nabla_{(c)} g=0, \quad T_{(c)}(X, J Y)=T_{(c)}(J X, Y),
$$

where $T_{(c)}$ is the torsion of $\nabla_{(c)}$.

In the case of an 1.c.K. manifold, it is easy to verify, using formulas of [10], [12], that one has

$$
\nabla_{(c) X} Y=\tilde{\nabla}_{X} Y+\frac{1}{2} \omega(X) Y-\frac{1}{2} \theta(X) J Y,
$$

and that the curvature of this connection is

$$
R_{(c)}(X, Y) Z=\tilde{R}(X, Y) Z-\frac{1}{2} d \theta(X, Y) J Z .
$$

If local complex coordinates are considered this formula becomes

$$
R_{(c) j k \bar{h}}^{i}=\tilde{R}_{j k \bar{h}}^{i}-\delta_{j}^{i} \partial_{\bar{h}} \omega_{k},
$$

and we see that this tensor has Kählerian symmetries iff $d^{\prime \prime} \omega^{\prime}=0$. Hence, by Remark (1) of $\$ 2$, we may state

Proposition 3.3. A compact l.c.K. manifold is Kähler iff the curvature $R_{(c)}$ has the symmetries of the curvature tensor of a Kähler manifold.

As a matter of fact, a stronger result is available. In order to write it down, let us introduce the unitary covariant curvature tensor

$$
R_{(c)}(X, Y, Z, W)=g\left(R_{(c)}(Z, W) Y, X\right)
$$

Then, we have

Proposition 3.4. Let $M$ be a connected l.c.K. manifold, which satisfies the following symmetry property

$$
R_{(c)}(X, Y, Z, W)=R_{(c)}(Z, W, X, Y) .
$$

Then, either $M$ is g.c.K. or $d \theta=0$ and then, if $M$ is compact, $M$ is Kähler.

Proof. As a consequence of (3.4) and (3.7) we obtain

$$
d \theta(X, Y) \Omega(Z, W)=d \theta(Z, W) \Omega(X, Y) .
$$

This implies $d \theta=\varphi \Omega$ and, by a differentiation, $d \varphi+\varphi \omega=0$. Hence, if $\varphi \neq 0$ everywhere, $\omega$ is exact and $M$ is g.c.K. On another side, if $\varphi\left(x_{0}\right)=0$ for some $x_{0} \in M$, then, by putting $\omega=d \tau / \tau$ in some neighbourhood of $x_{0}$, we obtain from $d \varphi+\varphi \omega=0$ that $\varphi=0$ on the whole neighbourhood. Since any other point 
$x \in M$ can be joined to $x_{0}$ by a chain of consecutively intersecting neighbourhoods, where $\omega$ is of the form $d \tau / \tau$, we see that $\varphi \equiv 0$, i.e., $d \theta=0$ and, with Remark (1) of $\S 2$, we are done.

COROllary 3.5. A compact l.c.K. manifold with $R_{(c)}=0$ is Kähler

Now, let us consider the unitary sectional curvature

$$
K_{(c)}(X, Y)=\frac{R_{(c)}(X, Y, X, Y)}{|X|^{2}|Y|^{2}-(g(X, Y))^{2}} .
$$

In view of (3.4), and over each neighbourhood $U_{\alpha}$, we have

$$
K_{(c)}(X, Y)=e^{-\sigma_{\alpha}} \tilde{K}_{\alpha}(X, Y)+\frac{1}{2} \frac{d \theta(X, Y) \Omega(X, Y)}{(g(X, Y))^{2}-|X|^{2}|Y|^{2}},
$$

where $\tilde{K}_{\alpha}(X, Y)$ is the sectional curvature of the Kähler metric $\tilde{g}_{\alpha}$. As a consequence of this formula, it is easy to see that the unitary sectional curvature depends, in fact, on the plane of $\{X, Y\}$ and not on these vectors themselves.

Proposition 3.6. Let $M$ be a connected l.c.K. manifold with pointwise constant unitary sectional curvature. Then, either $M$ is g.c.K. or $M$ has a vanishing first Chern class.

Proof. Let us introduce the following notation:

$$
\begin{gathered}
G(X, Y, Z, W)=g(X, Z) g(Y, W)-g(Y, Z) g(X, W), \\
R_{(s c)}(X, Y, Z, W)=\frac{1}{2}\left\{R_{(c)}(X, Y, Z, W)+R_{(c)}(Z, W, X, Y)\right\}, \\
-4 \Theta(X, Y, Z, W)=d \theta(X, Y) \Omega(Z, W)+d \theta(Z, W) \Omega(X, Y) .
\end{gathered}
$$

The hypothesis $K_{(c)}(X, Y)$-pointwise constant means

$$
R_{(s c)}(X, Y, X, Y)=\varphi G(X, Y, X, Y)
$$

for some real function $\varphi$ on $M . R_{(s c)}$ does not necessarily satisfy the Riemannian Bianchi identity.

However, that part of the proof of Proposition 1.2. of [3, Chapter V] which is not based on the Bianchi identity allows us to deduce

$$
R_{s c}(X, Y, Z, W)-R_{s c}(X, W, Y, Z)=\varphi\{G(X, Y, Z, W)-G(X, W, Y, Z)\}
$$

or, by computing the tensor $R_{(s c)}$ by means of (3.4):

$$
\begin{gathered}
\Theta(X, Y, Z, W)-\Theta(X, W, Y, Z)+e^{\sigma_{\alpha}}\left\{2 \tilde{R}_{\alpha}(X, Y, Z, W)+\tilde{R}_{\alpha}(X, Z, W, Y)\right\} \\
=\varphi\{G(X, Y, Z, W)-G(X, W, Y, Z)\} .
\end{gathered}
$$

Now, we shall explicitate (3.11) locally by putting $X=\partial / \partial z^{i}, Y=\partial / \partial \bar{z}^{j}, Z=$ $\partial / \partial z^{k}, W=\partial / \partial \bar{z}^{h}$. Since $\tilde{R}_{\alpha}$ is a Kählerian curvature, we obtain

$$
\frac{1}{2} \partial_{j} \omega_{i} g_{k \bar{h}}+\frac{1}{2} \partial_{\bar{h}} \omega_{k} g_{i j}+\frac{1}{2} \partial_{\bar{h}} \omega_{i} g_{k j}+\frac{1}{2} \partial_{j} \omega_{k} g_{i \bar{h}}+2 e^{\sigma_{\alpha}} \tilde{R}_{\alpha \overline{j k} \bar{h}}=-\varphi\left\{g_{i \bar{h}} g_{k \bar{j}}+g_{i j} g_{k \bar{h}}\right\} \text {. }
$$

By contracting here with $g^{\bar{h} k}$, this relation implies

$$
\frac{1}{2}(n+2) \partial_{j} \omega_{i}-2 \tilde{R}_{i j}=\psi g_{i j},
$$

where $\psi=-(n+1) \varphi-\frac{1}{2} g^{\bar{h} k} \partial_{\bar{h}} \omega_{k}$. 
Finally, this relation means

$$
\frac{1}{2}(n+2) d \theta+8 \pi C_{1}=2 \psi \Omega,
$$

where $C_{1}$ is a closed form defining the first Chern class of $M$ [3, Vol. II, p. 312].

By a differentiation, (3.12) provides $d \psi+\psi \omega=0$, whence, as in the case of the proof of Proposition 3.4, we must have either $\omega$ exact or $\psi=0$, i.e., either $M$ is g.c.K. or $C_{1}$ is exact by (3.12). Q.E.D.

ReMARKS. (1) If $C_{1}=0$ (i.e., the Kähler metrics $\tilde{g}_{\alpha}$ are Ricci flat), formula (3.12) proves in a similar manner that either $M$ is g.c.K. or $d \theta=0$ (and $M$ is Kähler, if compact).

(2) Various other results can be proven in a similar manner, e.g., if $M$ is a connected l.c.K. manifold whose local Kähler metrics $\tilde{g}_{\alpha}$ have vanishing holomorphic sectional curvature and whose unitary holomorphic sectional curvature is pointwise constant then either $M$ is g.c.K. or $d \theta=0$, etc. The stated result follows from the following consequence of (3.4):

$$
K_{(c)}(X, J X)=e^{-\sigma_{\alpha}} \tilde{K}_{\alpha}(X, J X)+d \theta(X, J X) / 2|X|^{2} .
$$

Let us note that, together with formulas in [12], (3.13) implies

$$
K_{(c)}(X, J X)=K(X, J X)+|\omega|^{2} / 4-\left(\omega^{2}(X)+\theta^{2}(X)\right) / 4|X|^{2} .
$$

4. I.c.K. manifolds are analytically irreducible. The present section is devoted to the proof of the fact that, if an 1.c.K. metric is locally analytically reducible then it is necessarily a Kähler metric.

The proof is a matter of a computation, and we prefer to do it in the following way.

By the local analytical reducibility of $g$ we understand the existence of such local complex coordinates $\left(z^{a}, z^{u}\right)$ such that $g$ has locally the form

$$
g_{a b}\left(z^{c}\right) d z^{a} d z^{b}+g_{u v}\left(z^{w}\right) d z^{u} d z^{v},
$$

where $a, b, c=1, \ldots, h ; u, v, w=h+1, \ldots, n ; 1 \leqslant h \leqslant n-1$. In other words, $M$ must have a pair of complementary orthogonal distributions $D, D^{\prime}$ which are analytic [9], integrable, and define foliations with respect to which $g$ is a bundlelike metric [6].

This pair $D, D^{\prime}$ can be defined by a pair of projectors $(P, Q)$ such that [9]

$$
\begin{gathered}
P^{2}=P, \quad Q^{2}=Q, \quad P Q=Q P=0, \quad P+Q=\mathrm{Id}, \\
g(P X, Q Y)=0, \quad P J=J P, \quad Q J=J Q .
\end{gathered}
$$

Namely, $P$ and $Q$ are the orthogonal projections of the tangent space of $M$ onto $D$ and $D^{\prime}$, respectively, and $D=\operatorname{im} P, D^{\prime}=\operatorname{im} Q$.

The integrability of $D, D^{\prime}$ means

$$
Q[P X, P Y]=0, \quad P[Q X, Q Y]=0 .
$$

In what concerns the analyticity we can use

Proposition 4.1. A complex distribution $D$ on a complex analytic manifold $M$ is an analytic distribution iff, for any vector field $X$ on $M$ and any vector field $Y$ of $D$, the vector field $[J X, Y]-J[X, Y]$ lies in $D$. 
This has been proven in [9] for a Kähler manifold $M$, but, since analyticity has a local character, it is true in the general case as well.

For $D$ and $D^{\prime}$ considered previously, this means

$$
Q\{[J X, P Y]-J[X, P Y]\}=0, \quad P\{[J X, Q Y]-J[X, Q Y]\}=0 .
$$

Finally, the conditions of $g$ being bundle-like are [9]

$$
\left(L_{P X} g\right)(Q Y, Q Z)=0, \quad\left(L_{Q X} g\right)(P Y, P Z)=0,
$$

where $L$ denotes the Lie derivative.

All is ready now for the proof of

Proposition 4.2. An l.c.K. manifold which is analytically reducible is a Kähler manifold.

Proof. By explicitating with the usual formula of the exterior differential, and in view of the conditions (4.1)-(4.4), we derive after a computation

$$
d \Omega(Q X, Q Y, P Z)=0, \quad d \Omega(P X, P Y, Q Z)=0 .
$$

Now, because of the l.c.K. condition $d \Omega=\omega \wedge \Omega$, these relations imply

$$
\omega(P Z) \Omega(Q X, Q Y)=0, \quad \omega(Q Z) \Omega(P X, P Y)=0,
$$

and this in turn implies $\omega=0$. Q.E.D.

REMARKS. (1) As already mentioned this means that a non-Kähler l.c.K. manifold must be irreducible.

(2) The analytical character of the reducibility is essential in Proposition 4.2, since e.g., the Hopf manifolds are non-Kähler and 1.c.K. and, yet, $C^{\infty}$-reducible [14].

5. Some examples of I.c.K. manifolds. The known list of examples of 1.c.K. manifolds which are not g.c.K. is not very large. It contains the Hopf manifolds [10], [14] and, more generally, it contains the flat principal $S^{1}$-bundles over Sasakian manifolds [14]. All these belong to the class of the l.c.K. manifolds with parallel Lee form [14] which, in the compact case, must have an odd first Betti number [2]. Finally, an 1.c.K. and non-g.c.K. metric on $T_{c}^{1} \times C^{n-1}$ has been constructed in [10].

In this section, we should like to discuss some concrete examples related to the above-mentioned ones.

Let us start by considering the Hopf fibrations

$$
p: S^{2 n-1} \rightarrow C P^{n-1}, \quad \pi: H^{n} \rightarrow C P^{n-1},
$$

where $H^{n}$ is the $n$-dimensional Hopf manifold $(n>1)$. We are referring to [3, Vol. II] for the description of (5.1), and recall only the essential facts.

Thus, if $\left\{t^{k}\right\}(k=1, \ldots, n)$ denote cartesian coordinates in the complex space $C^{n}$, then $S^{2 n-1}$ is defined by $\sum_{k=1}^{n} t^{k^{-k}}=1 . C P^{n-1}$ is the set of the equivalence classes of $C^{n}-\{0\}$ with respect to proportionality. Its points will be denoted by using homogeneous coordinates $\left[w^{1}: \cdots: w^{n}\right] . H^{n}=\left(C^{n}-\{0\}\right) / \Delta_{\lambda}$, where $\Delta_{\lambda}$ is the group generated by $z \mapsto \lambda z, z \in C^{n}-\{0\}, \lambda \in C,|\lambda| \neq 0,1$. We denote a corresponding point in $H^{n}$ by $\left\{z^{1}, \ldots, z^{n}\right\} . p$ is a principal circle bundle and $\pi$ is 
a principal $T_{c}^{1}$-bundle, where

$$
T_{c}^{1}=C / D, \quad D=\{2 \pi i a+(\ln \lambda) b / a, b \in \mathbf{Z}\} .
$$

If $U_{\alpha}=\left\{\left[w^{1}: \cdots: w^{n}\right] / w^{\alpha} \neq 0\right\}(\alpha=1, \ldots, n)$, then both $p$ and $\pi$ have local trivializations over each $U_{\alpha}$ and these are given, respectively, by

$$
\begin{aligned}
\left(t^{1}, \ldots, t^{n}\right) & \mapsto\left(\left[t^{1}: \cdots: t^{n}\right], t^{\alpha} /\left|t^{\alpha}\right|\right), \\
\left(\left[w^{1}: \cdots: w^{n}\right], \tau\right) & \mapsto\left(\frac{\tau\left|w^{\alpha}\right| w^{1}}{w^{\alpha} \sqrt{\sum_{1}^{n} \bar{w}^{k} w^{k}}}, \ldots, \frac{\tau\left|w^{\alpha}\right| w^{n}}{w^{\alpha} \sqrt{\sum_{1}^{n} w^{k} \bar{w}^{k}}}\right)
\end{aligned}
$$

( $\left.\tau=e^{i t} \in S^{1}\right)$, for the fibration $p$, and

$$
\begin{aligned}
\left\{z^{1}, \ldots, z^{n}\right\} & \mapsto\left(\left[z^{1}: \cdots: z^{n}\right], \ln z^{\alpha}\right), \\
\left(\left[w^{1}: \cdots: w^{n}\right], \zeta\right) & \mapsto\left\{\frac{w^{1}}{w^{\alpha}} e^{\zeta}, \ldots, \frac{w^{n}}{w^{\alpha}} e^{\zeta}\right\},
\end{aligned}
$$

( $\zeta \in T_{c}^{1}$ ) for the fibration $\pi$.

On every $U_{\alpha}$, we shall also use the nonhomogeneous coordinates $w_{(\alpha)}^{k}=w^{k} / w^{\alpha}$ $(k=1, \ldots, n)$.

Now, for use in the subsequent discussion, we shall consider the classical contact structure on $S^{2 n-1}$, which is defined by

$$
\theta=-\frac{\sqrt{-1}}{2} \sum_{k=1}^{n}\left(t^{k} d t^{k}-\bar{t}^{k} d t^{k}\right)
$$

and the 1.c.K. metric of $H^{n}$ given by [10]

$$
d s^{2}=\left(1 / \sum_{k=1}^{n} z^{k} \bar{z}^{k}\right) \sum_{j=1}^{n} d z^{j} d \bar{z}^{j} .
$$

The Lee form of this metric is

$$
\omega=\left(-1 / \sum_{k=1}^{n} z^{k} \bar{z}^{k}\right) \sum_{j=1}^{n}\left(z^{j} d \bar{z}^{j}+\bar{z}^{j} d z^{j}\right) .
$$

If we go over to the local coordinates provided by (5.2) and (5.3), and use nonhomogeneous coordinates, these elements become

$$
\begin{gathered}
\theta=-d t-\frac{\sqrt{-1}}{2} \frac{\sum_{k \neq \alpha}\left(w_{(\alpha)}^{k} d \bar{w}_{(\alpha)}^{k}-\bar{w}_{(\alpha)}^{k} d w_{(\alpha)}^{k}\right)}{1+\sum_{h \neq \alpha} w_{(\alpha)}^{h} \bar{w}_{(\alpha)}^{h}}, \\
d s^{2}=d \zeta d \bar{\zeta}+d \zeta \frac{\sum_{j \neq \alpha} w_{(\alpha)}^{j} d \bar{w}_{(\alpha)}^{j}}{1+\sum_{h \neq \alpha} w_{(\alpha)}^{h} \bar{w}_{(\alpha)}^{h}}+\frac{\sum_{j \neq \alpha} \bar{w}_{(\alpha)}^{j} d w_{(\alpha)}^{j}}{1+\sum_{h \neq \alpha} w_{(\alpha)}^{h} \bar{w}_{(\alpha)}^{h}} d \bar{\zeta} \\
+\frac{\sum_{1}^{n} d w_{(\alpha)}^{j} d \bar{w}_{(\alpha)}^{j}}{1+\sum_{h \neq \alpha} w_{(\alpha)}^{h} \bar{w}_{(\alpha)}^{h}}, \\
\omega=-d \zeta-d \bar{\zeta}-\frac{\sum_{j \neq \alpha}\left(w_{(\alpha)}^{j} d \bar{w}_{(\alpha)}^{j}+\bar{w}_{(\alpha)}^{j} d w_{(\alpha)}^{j}\right)}{1+\sum_{h \neq \alpha} w_{(\alpha)}^{h} \bar{w}_{(\alpha)}^{h}}
\end{gathered}
$$


Let us also recall [3] that $\pi$ is differentiably isomorphic to the product of the fibration $p$ and the trivial fibration of $S^{1}$ over a one-point space.

Now, if $i: M \rightarrow C P^{n-1}$ is an analytical immersion, an induced fibration $\sigma: N \rightarrow M, \sigma=i^{-1}(\pi)$ exists, and $N$ will be called the induced Hopf manifold over $M$.

Proposition 5.1. (i) Every induced Hopf manifold has an induced l.c.K. metric which is not g.c.K. (ii) Every induced Hopf manifold is the product of $S^{1}$ with a regular Sasakian manifold.

Proof. Let us use the prior notation and take the corresponding pullback diagram

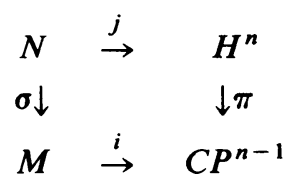

Clearly, $j$ is an immersion, and $N$ has the l.c.K. metric $j^{*}\left(d s^{2}\right)$ with Lee form $j^{*} \omega$, where $d s^{2}$ and $\omega$ are defined by (5.5), (5.6), respectively. It follows from (5.9) that $j^{*} \omega$ is not exact, and this proves (i).

In order to prove (ii), we take the fibration $\nu=i^{-1}(p): L \rightarrow M$ and the corresponding immersion $h: L \rightarrow S^{2 n-1}$. Then $h^{*} \theta$, with $\theta$ of (5.4), (5.5), defines a regular Sasakian structure on $L$. Since $\pi$ is the product of $p$ with $S^{1} \rightarrow$ \{point , we see that $N \approx L \times S^{1}$. Q.E.D.

COROllary 5.2. (i) If $M$ is a compact Hodge manifold, a $T_{c}^{1}$-principal fibration $\sigma: N \rightarrow M$ exists, with a compact l.c.K., not g.c.K., total space $N$. (ii) The corresponding Betti numbers are related by

$$
b_{h}(N)=b_{h}(M)+b_{h-1}(M)-b_{h-2}(M)-b_{h-3}(M),
$$

$(h=0, \ldots, n-1)$ and by the corresponding Poincaré duals.

(See Theorem 36.2 of [7] for (ii).)

We put down the relations (5.10) in order to point out that the relation $b_{2}(N) \neq 0$ is possible. Indeed, if $M$ is an algebraic $T_{c}^{1}$, we get, by $(5.10), b_{2}(N)=2$. This is interesting because of the fact that, by Corollary 2.2, an induced Hopf manifold $N$ never has a Kähler metric.

Furthermore, we should like to make the remark that the induced Hopf manifolds must have a parallel Lee form. Indeed, since $N \approx L \times S^{1}$ is induced by $H \approx S^{2 n-1} \times S^{1}$, this assertion follows from Theorem (4.1) of [14].

\section{REFERENCES}

1. T. Kashiwada, Some properties of locally conformal Kähler manifolds, Hokkaido Math. J. 8 (1979), 191-198.

2. T. Kashiwada and S. Sato, On harmonic forms in a compact locally conformal Kähler manifold with parallel Lee form (preprint).

3. S. Kobayashi and K. Nomizu, Foundations of differential geometry. I, II, Interscience, New York, $1963,1969$.

4. K. Kodaira, On the structure of compact complex analytic surfaces, Amer. J. Math. 86 (1964), 751-798. 
5. Y. Matsushima, Holomorphic vector fields on compact Kähler manifolds, CBMS Regional Conf. Ser. in Math., no. 7, Amer. Math. Soc., Providence, R. I., 1971.

6. B. Reinhart, Foliated manifolds with bundle-like metrics, Ann. of Math. (2) 69 (1959), 119-131.

7. S. Sasaki, Almost contact manifolds. I, II, III, Math. Inst. Tôhoku Univ., 1965, 1967, 1968.

8. J. A. Schouten, Ricci-calculus, Springer-Verlag, Berlin, 1954.

9. I. Vaisman, On the analytic distributions and foliations of a Kähler manifold. Proc. Amer. Math. Soc. 58 (1976), 221-228.

10. , On locally conformal almost Kähler manifolds, Israel J. Math. 24 (1976), 338-351.

11., A theorem on compact locally conformal Kähler manifolds, Proc. Amer. Math. Soc. 75 (1979), 279-283.

12. , Some curvature properties of locally conformal Kähler manifolds, Trans. Amer. Math. Soc. 259 (1980), 439-447.

13. __ A geometric condition for an l.c.K. manifold to be Kähler, Geom. Dedicata (to appear).

14. __ Locally conformal Kähler manifolds with parallel Lee form, Rend. Mat. (2) 12 (1979), 265-284.

Department of Mathematics, University of Haifa, Haifa, IsRael 